\title{
Near Threshold Graphs
}

\author{
Steve Kirkland* \\ Department of Mathematics and Statistics \\ University of Regina \\ Regina, Saskatchewan, Canada S4S 0A2
}

Submitted: Feb 3, 2009; Accepted: Mar 16, 2009; Published: Mar 25, 2009

Mathematics Subject Classification: 05C50, 15A18

\begin{abstract}
A conjecture of Grone and Merris states that for any graph $G$, its Laplacian spectrum, $\Lambda(G)$, is majorized by its conjugate degree sequence, $D^{*}(G)$. That conjecture prompts an investigation of the relationship between $\Lambda(G)$ and $D^{*}(G)$, and Merris has characterized the graphs $G$ for which the multisets $\Lambda(G)$ and $D^{*}(G)$ are equal. In this paper, we provide a constructive characterization of the graphs $G$ for which $\Lambda(G)$ and $D^{*}(G)$ share all but two elements.
\end{abstract}

\section{Introduction}

Let $G$ be a simple, undirected graph on $n$ vertices labeled $1, \ldots, n$. The Laplacian matrix for $G$, which we denote by $L(G)$ is the matrix given by $L(G)=D-A$, where $A$ is the $(0,1)$ adjacency matrix of $G$, and where $D$ is the diagonal matrix of vertex degrees. Evidently $L(G)$ is a symmetric matrix, and it is not difficult to determine that it is positive semi-definite, with the all ones vector, $\mathbf{1}$, as a null vector. In fact it turns out that the nullity of $L(G)$ coincides with the number of connected components of $G$. For these, and other properties of Laplacian matrices, we refer the reader to the surveys [10] and [13]. As can be seen from those two surveys, there is a wealth of literature on Laplacian matrices for graphs, much of it focusing on the interplay between the combinatorial properties of graphs and the eigenvalue and eigenvector properties of their corresponding Laplacian matrices.

Suppose that a graph $G$ on $n$ vertices has degree sequence $\delta \equiv d_{1} \leq \ldots \leq d_{n} \equiv \Delta$, and Laplacian eigenvalues $0=\lambda_{1} \leq \ldots \leq \lambda_{n}$. For each $j=1, \ldots, n$, we set $d_{j}^{*}=\left|\left\{i \mid d_{i} \geq j\right\}\right|$; evidently $d_{1}^{*} \geq d_{2}^{*} \geq \ldots \geq d_{n}^{*}$, and $d_{j}^{*}=0$ if either $j<\delta$ or $j>\Delta$. The entire sequence $d_{1}^{*}, \ldots, d_{n}^{*}$ is known as the conjugate degree sequence of $G$. Henceforth, we let $\Lambda(G)$ and $D^{*}(G)$ denote the multisets consisting of the Laplacian eigenvalues of $G$, and the conjugate

${ }^{*}$ Research partially supported by NSERC under grant number OGP0138251. 
degree sequence of $G$, respectively. In the interests of clarity, we will occasionally write $\lambda_{i}(G)$ or $d_{j}^{*}(G)$ to emphasize the dependence on $G$ of a particular eigenvalue or element of the conjugate degree sequence.

Recall that given two vectors of real numbers, both listed in nonincreasing order, $x=\left[x_{1}, \ldots, x_{n}\right]$ and $y=\left[y_{1}, \ldots, y_{n}\right]$ we say that $x$ majorizes $y$, and write $x \succeq y$, if we have $\sum_{i=1}^{k} x_{i} \geq \sum_{i=1}^{k} y_{i}$ for each $k=1, \ldots, n-1$, and $\sum_{i=1}^{n} x_{i}=\sum_{i=1}^{n} y_{i}$. A result attributed to Horn, and also to Schur, asserts that $x \succeq y$ if and only if there is a symmetric matrix of order $n$ with spectrum $x_{1}, \ldots, x_{n}$ and diagonal entries $y_{1}, \ldots, y_{n}$. From that fact, Grone and Merris [4] observe that the Laplacian spectrum for a graph majorizes its degree sequence. Further, in that paper they also conjecture that the conjugate degree sequence for a graph, in turn, majorizes its Laplacian spectrum. That conjecture has come to be known as the Grone-Merris conjecture, and it has been verified for several classes of graphs; see [1] and [14], for example.

In view of the Grone-Merris conjecture, it is natural to further explore the relationship between $D^{*}(G)$ and $\Lambda(G)$. Indeed in [9], Merris does exactly that, characterizing the graphs $G$ such that $D^{*}(G)=\Lambda(G)$. It turns out that the class of graphs for which the Laplacian spectrum and the conjugate degree sequence coincide is exactly the class of threshold graphs - i.e., those graphs having no induced subgraphs equal to either $P_{4}, C_{4}$, or $2 K_{2}$. We note that Laplacian matrices for threshold graphs (which are referred to as degree maximal graphs in [9]) have been discussed from a variety of perspectives; see [2], [5] and [8] for a sampling of results of that type.

In this paper, we pursue a line of inquiry that is inspired by [9] by looking at graphs for which $D^{*}(G)$ and $\Lambda(G)$ share a large number of elements. In order that we can be more precise, we introduce some terminology. Given a graph $G$ on $n$ vertices, we say that $\Lambda(G)$ and $D^{*}(G)$ agree in $k$ places if there is a multiset $S$ of cardinality $k$ and indices $i_{1}, \ldots, i_{n-k}$ and $j_{1}, \ldots, j_{n-k}$ such that $\Lambda(G)=S \cup\left\{\lambda_{i_{1}}, \ldots, \lambda_{i_{n-k}}\right\}, D^{*}(G)=S \cup\left\{d_{j_{1}}^{*}, \ldots, d_{j_{n-k}}^{*}\right\}$, where $\left\{\lambda_{i_{1}}, \ldots, \lambda_{i_{n-k}}\right\} \cap\left\{d_{j_{1}}^{*}, \ldots, d_{j_{n-k}}^{*}\right\}=\emptyset$. Observe that if that condition holds, then necessarily $\sum_{p=1}^{n-k} \lambda_{i_{p}}=\sum_{q=1}^{n-k} d_{j_{q}}^{*}$. For a graph $G$ on $n$ vertices, we thus see that $D^{*}(G)$ and $\Lambda(G)$ agree in $n$ places if and only if $G$ is a threshold graph; further, it is not difficult to see that $D^{*}(G)$ and $\Lambda(G)$ cannot agree in $n-1$ places. In this paper, we deal with the case that $D^{*}(G)$ and $\Lambda(G)$ agree in $n-2$ places. Henceforth, we say that the graph $G$ is a near threshold graph (or NT graph for short) if $\Lambda(G)$ and $D^{*}(G)$ agree in $n-2$ places. We note in passing that it straightforward to show that a graph $G$ is an NT graph if and only if its complement, $\bar{G}$, is an NT graph.

Example 1.1 Of the graphs on 4 vertices, the only ones that are not threshold graphs are $C_{4}, P_{4}$, and $2 K_{2}$. Observe that $\Lambda\left(C_{4}\right)=\left\{0,2^{(2)}, 4\right\}, D^{*}\left(C_{4}\right)=\left\{4^{(2)}, 0^{(2)}\right\}, \Lambda\left(P_{4}\right)=$ $\{0,2-\sqrt{2}, 2,2+\sqrt{2}\}, D^{*}\left(P_{4}\right)=\left\{4,2,0^{(2)}\right\}$, and $\Lambda\left(2 K_{2}\right)=\left\{0^{(2)}, 2^{(2)}\right\}, D^{*}\left(2 K_{2}\right)=\left\{4,0^{(3)}\right\}$ (here, as elsewhere we use a superscript in parentheses to denote the multiplicity of an element in a multiset). Thus we find that each of $C_{4}, P_{4}$ and $2 K_{2}$ is an NT graph.

In sections 2 and 3, we provide a constructive characterization of the class of NT graphs. Throughout, we will assume familiarity with basic results and techniques from 
graph theory and matrix theory. We refer the reader to [12] and [6] respectively for background in those areas.

\section{The disconnected case}

Suppose that $G$ is a graph on $n$ vertices, $m$ of which are isolated. Then $G$ can be written as $G=H \cup O_{m}$, where $O_{m}$ denotes the empty graph on $m$ vertices. It follows that $\Lambda(G)=\Lambda(H) \cup\left\{0^{(m)}\right\}$, while $D^{*}(G)=D^{*}(H) \cup\left\{0^{(m)}\right\}$. In this setting, we see that $G$ is an NT graph if and only if $H$ is an NT graph. We summarize this as the following.

Proposition 2.1 Let $G$ be a graph on $n$ vertices $m$ isolated vertices. Then $G$ is an $N T$ graph if and only if $G=H \cup O_{m}$, where $H$ is an NT graph on $n-m$ vertices.

Our next result with be useful in the sequel.

Lemma 2.2 Suppose that $G$ is a disconnected graph with no isolated vertices, say $G=$ $\cup_{i=1}^{m} H_{i}$, where each $H_{i}$ is a connected graph on $n_{i}$ vertices, and where $n_{1} \geq \ldots \geq n_{m} \geq 2$. If $G$ is an NT graph, then $m=2$ and $n_{2}=2$, so that $G=H_{1} \cup K_{2}$.

Proof: We have 0 as an eigenvalue of $G$ of multiplicity $m$, while $0=d_{n_{1}}^{*}=d_{n_{1}+1}^{*}=\ldots=$ $d_{\sum_{i=1}^{*} n_{i}}^{*}$, so that $D^{*}(G)$ contains 0 with multiplicity at least $1+\sum_{i=2}^{m} n_{i}$. Since there are indices $i_{1}, i_{2}, j_{1}, j_{2}$ such that $\lambda_{i_{1}}+\lambda_{i_{2}}=d_{j_{1}}^{*}+d_{j_{2}}^{*}$, and $\left\{\lambda_{i_{1}}, \lambda_{i_{2}}\right\} \cap\left\{d_{j_{1}}^{*}+d_{j_{2}}^{*}\right\}=\emptyset$, we find that at most one of $d_{j_{1}}^{*}, d_{j_{2}}^{*}$ is zero. Hence $\sum_{i=2}^{m} n_{i} \leq m$. Consequently, we have $0 \leq \sum_{i=2}^{m}\left(n_{i}-2\right) \leq m-2 m+2=2-m$. We find that necessarily $m=2$ and $n_{2}=2$, from which the conclusion follows.

Recall that a vertex of a graph $G$ is dominant if it is adjacent to all other vertices of $G$. Recall also that for two graphs $G_{1}, G_{2}$, their join, denoted $G_{1} \vee G_{2}$, is the graph formed from the union of $G_{1}$ and $G_{2}$ by adding all possible edges between vertices in $G_{1}$ and vertices in $G_{2}$. Here is the main result of this section.

Theorem 2.3 Suppose that $G$ is a disconnected graph on $n \geq 4$ vertices, with no isolated vertices. Then $G$ is an NT graph if and only if one of the following holds:

a) $G=H \cup K_{2}$, where $H$ is a connected threshold graph;

b) $G=\left(K_{2} \vee H_{0}\right) \cup K_{2}$, where $H_{0}$ is an NT graph with no isolated vertices and no dominant vertices.

Proof: First we suppose that $G$ is a disconnected NT graph with no isolated vertices. From Lemma 2.2 we find that necessarily $G=H \cup K_{2}$ for some connected graph $H$ on at least two vertices. In particular, $\Lambda(G)=\{0,2\} \cup \Lambda(H), d_{1}^{*}(G)=n, d_{n-1}^{*}(G)=d_{n}^{*}(G)=0$, and $d_{i}^{*}(G)=d_{i}^{*}(H), i=2, \ldots, n-2$. Since the eigenvalues of $G$ are bounded above by $n-2$, we find that $n \notin \Lambda(G)$; note also that 0 has multiplicity at least 3 in $D^{*}(G)$ and multiplicity 2 in $\Lambda(G)$. Since $G$ is an NT graph, it follows that there is a multiset $S$ 
of cardinality $n-2$, and indices $i_{1}, i_{2}$ such that $\Lambda(G)=S \cup\left\{\lambda_{i_{1}}, \lambda_{i_{2}}\right\}$, and $D^{*}(G)=$ $S \cup\{0, n\}$. Indeed, it must be the case that $S=\left\{0, d_{2}^{*}(H), d_{3}^{*}(H), \ldots, d_{n-2}^{*}(H)\right\}$. Further, since $\operatorname{trace}(L(G))=\sum_{i=1}^{n} d_{i}^{*}(G)$, we find that $\lambda_{i_{1}}+\lambda_{i_{2}}=n$.

Taking $\lambda_{i_{1}} \leq \lambda_{i_{2}}$, and recalling that $\lambda_{i_{2}} \leq n-2$, we find that for some $2 \leq x \leq \frac{n}{2}$, the ordered pair $\left(\lambda_{i_{1}}, \lambda_{i_{2}}\right)$ coincides with $(x, n-x)$. Since $S$ has exactly two zeros, we see that necessarily $d_{n-3}^{*}(G)=d_{n-3}^{*}(H)$ must be positive, so that $H$ has at least one dominant vertex.

Suppose first that $\left(\lambda_{i_{1}}, \lambda_{i_{2}}\right)=(2, n-2)$. Then we have both $\Lambda(G)=\Lambda(H) \cup\{0,2\}$ and $\Lambda(G)=\left\{0, d_{2}^{*}(H), d_{3}^{*}(H), \ldots, d_{n-2}^{*}(H)\right\} \cup\{2, n-2\}$. We then deduce that $\Lambda(H)=$ $\left\{n-2, d_{2}^{*}(H), d_{3}^{*}(H), \ldots, d_{n-2}^{*}(H)\right\}=D^{*}(H)$. Consequently, we find that $H$ must be a connected threshold graph, so that condition a) is satisfied.

Next, suppose that $\left(\lambda_{i_{1}}, \lambda_{i_{2}}\right)=(x, n-x)$ for some $2<x \leq \frac{n}{2}$. Note that the smallest positive element of $D^{*}(G)$ is $d_{n-3}^{*}(G)$; since $2 \in S$, it now follows that $d_{n-3}^{*}(H) \leq 2$. As $H$ has a dominant vertex, we find that either $d_{n-3}^{*}(H)=1$ or $d_{n-3}^{*}(H)=2$.

If $d_{n-3}^{*}(H)=1$ then necessarily $1 \in \Lambda(H)$ as well. It follows that $H$ can be written as $H=K_{1} \vee\left(H_{1} \cup \ldots \cup H_{\ell}\right)$ for some $\ell \geq 2$, where each $H_{i}$ is a connected graph (possibly consisting of just a single vertex). For each $i=1, \ldots, \ell$, let $m_{i}$ be the number of vertices in $H_{i}$; note that $\sum_{i=1}^{\ell} m_{i}=n-3$. Without loss of generality, we take $m_{1} \geq \ldots \geq m_{\ell}$. Note that, apart from the dominant vertex in $H$, the degree of any other vertex of $H$ is at most $m_{1}$, and that $m_{1}=n-3-\sum_{i=2}^{\ell} m_{i} \leq n-3-(\ell-1)=n-\ell-2$. In particular, we find that $d_{n-\ell-1}^{*}(H)=1$, and hence that $d_{j}^{*}(H)=1$, for $j=n-\ell-1, \ldots, n-3$. From the structure of $H$, we find that 1 is an eigenvalue of $H$ of multiplicity $\ell-1$ (and hence 1 is an eigenvalue of $G$ with the same multiplicity). Consequently, we find that $d_{n-\ell-2}^{*}(H)>1$, and since 2 is an eigenvalue of $G$, we deduce that in fact $d_{n-\ell-2}^{*}(H)=2$. From the fact that $m_{1} \leq n-\ell-2$, we find that $m_{i}=1, i=2, \ldots, \ell$, and that $H_{1}$ can be written as $K_{1} \vee H_{0}$ for some graph $H_{0}$ on $n-\ell-3$ vertices, where $H_{0}$ does not have a dominant vertex. Thus we find that $H$ can be written as $H=K_{1} \vee\left(\left(K_{1} \vee\right.\right.$ $\left.\left.H_{0}\right) \cup O_{\ell-1}\right)$. We may now write $D^{*}(G)$ and $\Lambda(G)$ in terms of $D^{*}\left(H_{0}\right)$ and $\Lambda\left(H_{0}\right)$ as follows: $D^{*}(G)=\left\{0^{(3)}, 1^{(\ell-1)}, 2, d_{1}^{*}\left(H_{0}\right)+2, \ldots, d_{n-\ell-5}^{*}\left(H_{0}\right)+2, n-\ell-1, n\right\} ; \Lambda(G)=$ $\left\{0^{(2)}, 1^{(\ell-1)}, 2, \lambda_{2}\left(H_{0}\right)+2, \ldots, \lambda_{n-\ell-3}\left(H_{0}\right)+2, n-\ell-1, n-2\right\}$. If $G$ is an NT graph, then it must be the case that the multisets $A=\left\{0, d_{1}^{*}\left(H_{0}\right)+2, \ldots, d_{n-\ell-5}^{*}\left(H_{0}\right)+2, n\right\}$ and $B=\left\{\lambda_{2}\left(H_{0}\right)+2, \ldots, \lambda_{n-\ell-3}\left(H_{0}\right)+2, n-2\right\}$ have the property that $|A \backslash B|=|B \backslash A|=2$. Since $0, n \notin B$, and $n-2 \notin A$, it now follows that $\left(\lambda_{i_{1}}, \lambda_{i_{2}}\right)=(2, n-2)$, contrary to assumption.

Next, we consider the case that $\left(\lambda_{i_{1}}, \lambda_{i_{2}}\right)=(x, n-x)$ for some $2<x \leq \frac{n}{2}$ and that $d_{n-3}^{*}(H)=2$. Thus we find that $H=K_{2} \vee H_{0}$ for some graph $H_{0}$ with no dominant vertices. Then we may write $\Lambda(G)$ and $D^{*}(G)$ as follows: $\Lambda(G)=\left\{0^{(2)}, 2, \lambda_{2}\left(H_{0}\right)+\right.$ $\left.2, \ldots, \lambda_{n-4}\left(H_{0}\right)+2,(n-2)^{(2)}\right\} ; D^{*}(G)=\left\{0^{(3)}, d_{1}^{*}\left(H_{0}\right)+2, \ldots, d_{n-5}^{*}\left(H_{0}\right)+2, n-2, n\right\}$. Since $G$ is an NT graph, we thus find that there are indices $i_{1}, i_{2}$ such that $2<\lambda_{i_{1}}\left(H_{0}\right)+2 \leq$ $\lambda_{i_{2}}\left(H_{0}\right)+2<n-2, \lambda_{i_{1}}\left(H_{0}\right)+2+\lambda_{i_{2}}\left(H_{0}\right)+2=n$, and $\left\{d_{1}^{*}\left(H_{0}\right)+2, \ldots, d_{n-5}^{*}\left(H_{0}\right)+2\right\}=$ $\left\{2, \lambda_{2}\left(H_{0}\right)+2, \ldots, \lambda_{n-4}\left(H_{0}\right)+2, n-2\right\} \backslash\left\{\lambda_{i_{1}}\left(H_{0}\right)+2, \lambda_{i_{2}}\left(H_{0}\right)+2\right\}$. Observe that since $H_{0}$ has no dominant vertices, $d_{n-5}^{*}\left(H_{0}\right)+2=2$; it must also be the case that $d_{1}^{*}\left(H_{0}\right)+2=n-2$, from which we conclude that $H_{0}$ has no isolated vertices. Consequently, we find that 
$D^{*}\left(H_{0}\right)$ and $\Lambda\left(H_{0}\right)$ agree in $n-6$ places, so that $H_{0}$ is an NT graph with no isolated vertices and no dominant vertices. Thus condition b) holds.

Finally, it is straightforward to determine that if either of a) or b) holds, then $G$ must be an NT graph.

We close this section with two examples.

Example 2.4 We now illustrate the construction of Theorem 2.3 a). Let $G=K_{1,3} \cup K_{2}$; note that $\Lambda(G)=\left\{0^{(2)}, 1^{(2)}, 2,4\right\}$, while $D^{*}(G)=\left\{0^{(3)}, 1^{(2)}, 6\right\}$. Letting $S=\left\{0^{(2)}, 1^{(2)}\right\}$, we have $\Lambda(G)=S \cup\{2,4\}$ and $D^{*}(G)=\{0,6\}$.

Example 2.5 Here we illustrate the construction of Theorem $2.3 \mathrm{~b}$ ). Consider the graph $G=\left(K_{2} \vee P_{4}\right) \cup K_{2}$. We have $\Lambda(G)=\left\{0^{(2)}, 2,4-\sqrt{2}, 4,4+\sqrt{2}, 6^{(2)}\right\}$, while $D^{*}(G)=$ $\left\{0^{(3)}, 2,4,6^{(2)}, 8\right\}$. Letting $S=\left\{0^{(2)}, 2,4,6^{(2)}\right\}$, we see that $\Lambda(G)=S \cup\{4-\sqrt{2}, 4+\sqrt{2}\}$, and $D^{*}(G)=S \cup\{0,8\}$.

\section{The connected case}

Suppose that $G$ is a connected NT graph on $n$ vertices with maximum degree $\Delta$. We have $d_{\Delta}^{*}>0$ and $d_{\Delta+1}^{*}=0, \ldots, d_{n}^{*}=0$, so that the sequence $D^{*}(G)$ contains exactly $n-\Delta$ zeros. Since $G$ is connected, $\Lambda(G)$ contains exactly one zero. Thus, the set $S=\Lambda(G) \cap D^{*}(G)$ contains at most one zero and at least $n-\Delta-1$ zeros, so that necessarily $n-\Delta-1 \leq 1$. Hence, $\Delta \geq n-2$.

Our next result deals with the possibility that $\Delta=n-1$.

Proposition 3.1 Let $G$ be a graph on $n$ vertices having $m \geq 1$ vertices of degree $n-1$. Then $G$ is an NT graph if and only if $G=K_{m} \vee H$, where $H$ is an NT graph on $n-m$ vertices.

Proof: Since $G$ has $m$ vertices of degree $n-1$, we find that $\bar{G}$ has $m$ isolated vertices. The conclusion now follows by appealing to Proposition 2.1, and the fact that $G$ is an NT graph if and only if $\bar{G}$ is an NT graph.

A graph $G$ on $n$ vertices that has $n$ as an eigenvalue with multiplicity $m$ can be written as a join of $m+1$ graphs of smaller order; see [11] for further information on the Laplacian spectrum of a join of graphs.

The following result provides some useful information.

Lemma 3.2 Suppose that $G$ is a connected $N T$ graph on $n \geq 4$ vertices with maximum degree $n-2$ and minimum degree $\delta$. Then there are distinct indices $i_{1}, i_{2}$ such that $\lambda_{i_{1}}+\lambda_{i_{2}}=n, \Lambda(G) \backslash\left\{\lambda_{i_{1}}, \lambda_{i_{2}}\right\}=D^{*}(G) \backslash\{0, n\}$, and $\left\{\lambda_{i_{1}}, \lambda_{i_{2}}\right\} \cap\{0, n\}=\emptyset$. 
Proof: First, note that since $G$ is connected, it has exactly one zero eigenvalue. Since the maximum degree is $n-2$, we find that $D^{*}(G)$ contains at least two zero elements. Since $G$ is an NT graph, it then follows that there are distinct indices $i_{1}, i_{2}$ and another index $j_{1}$ such that $\lambda_{i_{1}}+\lambda_{i_{2}}=0+d_{j_{1}}^{*}, \Lambda(G) \backslash\left\{\lambda_{i_{1}}, \lambda_{i_{2}}\right\}=D^{*}(G) \backslash\left\{0, d_{j_{1}}^{*}\right\}$, and $\left\{\lambda_{i_{1}}, \lambda_{i_{2}}\right\} \cap\left\{0, d_{j_{1}}^{*}\right\}=\emptyset$. We claim that $d_{j_{1}}^{*}=n$.

We have $d_{i}^{*}=n, i=1, \ldots, \delta$, and $d_{\delta+1}^{*}<n$. Since $G$ is an NT graph, it must then have $n$ as an eigenvalue of multiplicity $\delta-1$ or $\delta$. If the multiplicity of $n$ as an eigenvalue of $G$ is $\delta$, then $G$ can be written as a join of $\delta+1$ graphs, say $G=H_{1} \vee \ldots \vee H_{\delta+1}$. Since the minimum degree of $G$ is $\delta$, it follows that at most one of the graphs $H_{1}, \ldots, H_{\delta+1}$, say $H_{1}$, has more than one vertex. In that case, we can write $G=H_{1} \vee K_{\delta}$, which contradicts the hypothesis that $G$ has maximum degree $n-2$.

We conclude that $G$ has $n$ as an eigenvalue of multiplicity $\delta-1$, from which it follows that $d_{j_{1}}^{*}=n$.

Next, we describe the structure of connected NT graphs with minimum degree 2 and no dominant vertices.

Theorem 3.3 Let $G$ be a connected graph on $n \geq 4$ vertices with minimum degree $\delta \geq 2$ and maximum degree $n-2$. Then $G$ is an NT graph if and only if one of the following holds:

a) $G=G_{1} \vee O_{2}$ for some disconnected threshold graph $G_{1}$ on $n-2$ vertices;

b) there is a NT graph $H$ on $n-4$ vertices with no isolated vertices and no dominant vertices such that $\mathrm{G}=\left(\mathrm{O}_{2} \cup \mathrm{H}\right) \vee \mathrm{O}_{2}$.

Proof: Suppose that $G$ is an NT graph. Since $\delta \geq 2$, it follows that $n$ is an eigenvalue of $G$ of multiplicity at least $\delta-1$. Consequently $\bar{G}$ is a disconnected graph. Further, since the maximum degree of $G$ is $n-2$, we see that $\bar{G}$ has no isolated vertices. Applying Theorem 2.3 to $\bar{G}$, we find that $\bar{G}$ is an NT graph only if either $\bar{G}=H \cup K_{2}$, where $H$ is a connected threshold graph, or $\bar{G}=\left(K_{2} \vee H_{0}\right) \cup K_{2}$, where $H_{0}$ is an NT graph with no isolated vertices and no dominant vertices. The constructions a) and b) for $G$ now follows upon noting that $G$ is an NT graph only if $\bar{G}$ is an NT graph.

The converse is straightforward.

The next lemma identifies some of the spectral structure for NT graphs.

Lemma 3.4 Suppose that $G$ is a connected $N T$ graph on $n \geq 4$ vertices with minimum degree 1 and maximum degree $n-2$. Then $1<\lambda_{2}<2, n-1<\lambda_{n}<n, \lambda_{2}+\lambda_{n}=n$, and for each $i=1,3,4, \ldots, n-1$, we have $\lambda_{i}=d_{n-i+1}^{*}$.

Proof: From the hypotheses on the minimum and maximum degrees, we find that $d_{1}^{*}=$ $n, d_{n-1}^{*}=0, d_{n}^{*}=0, d_{2}^{*} \leq n-1$ and $d_{n-2}^{*} \geq 1$.

We claim that $n$ is not an eigenvalue of $G$. To see this, suppose to the contrary that $n$ is an eigenvalue. Then $G$ can be written as a join, say $G=H_{1} \vee H_{2}$. Since the minimum degree of $G$ is 1 , we see that one of $H_{1}$ and $H_{2}$ consists of a single vertex. But in that 
case, $G$ must have a vertex of degree $n-1$, contrary to the hypothesis. Hence $n$ is not an eigenvalue of $G$.

It now follows from the claim, and the fact that $D^{*}(G)$ contains two zeros while $\Lambda(G)$ contains only one zero, that there are indices $i, j$ such that $\lambda_{i}+\lambda_{j}=n, D^{*}(G) \backslash\{0, n\}=$ $\Lambda(G) \backslash\left\{\lambda_{i}, \lambda_{j}\right\}$, and $\{0, n\} \cap\left\{\lambda_{i}, \lambda_{j}\right\}=\emptyset$. A result in [7] asserts that for any connected graph $H$ with a cut vertex, we have $\lambda_{2}(H) \leq 1$, with equality if and only if $H$ has a dominant vertex. From the fact that the minimum degree of $G$ is 1 and there is no vertex of degree $n-1$, we thus find that $0<\lambda_{2}(G)<1$. Also, we have $n>\lambda_{n}(G)>n-2+1$, the rightmost strict inequality following from the fact that for a connected graph with maximum degree $\Delta, \lambda_{n}(G) \geq \Delta+1$, with equality only if $\Delta=n-1$ (see [4]). Hence $\lambda_{2}(G)$ and $\lambda_{n}(G)$ are non-integer eigenvalues of $G$, and the conclusion now follows.

Henceforth we consider a connected NT graph $G$ on $n$ vertices with minimum degree 1 , maximum degree $n-2, p$ vertices of degree 1 and $q$ vertices of degree $n-2$. The following lemma describes some of the structure for such a graph.

Lemma 3.5 Let $G$ be a connected $N T$ graph on $n \geq 4$ vertices with minimum degree 1 , maximum degree $n-2, p$ vertices of degree 1 and $q$ vertices of degree $n-2$.

a) If $G$ has two or more pendant vertices with a common neighbour, then $q=1$.

b) If $p \geq 3$, then $q=1$.

c) If $p=2$, then either $q=1$ or $G=P_{4}$.

Proof: We begin by noting that since $G$ has $p$ pendant vertices, we have $d_{2}^{*}=n-p=\lambda_{n-1}$, and since $G$ has $q$ vertices of degree $n-2$, we have $d_{n-2}^{*}=q=\lambda_{3}$.

a) If $G$ has two pendant vertices with a common neighbour, it follows readily that 1 is an eigenvalue for $G$. Since $\lambda_{1}=0, \lambda_{2}<1$, and all remaining eigenvalues, save for $\lambda_{n}$, are integers, it follows that in fact $\lambda_{3}=1$. Hence $q=1$.

b) Note that each vertex of degree $n-2$ is non-adjacent to at most one pendant vertex. Hence, if $p \geq 3$ then some vertex of degree $n-2$ is adjacent to at least $p-1 \geq 2$ vertices, and so from a) we find that $q=1$.

c) Suppose that $p=2$. If $q \geq 2$, then from a), the two pendant vertices cannot have a common neighbour. In particular, since each vertex of degree $n-2$ is not adjacent to at most one of the pendant vertices, we find that there can be at most two such vertices of degree $n-2$. Thus we conclude that $q=1$ or $q=2$.

Suppose that we are in the case that $q=2$. As above, we see that the two pendant vertices are adjacent to different vertices of degree $n-2$. If $n=4$, we find that $G=P_{4}$, as desired. Suppose now that $n \geq 5$. It follows that the Laplacian matrix for $G$ can be written as

$$
L=\left[\begin{array}{cc|cc|c}
1 & 0 & -1 & 0 & 0^{T} \\
0 & 1 & 0 & -1 & 0^{T} \\
\hline-1 & 0 & n-2 & -1 & -\mathbf{1}^{T} \\
0 & -1 & -1 & n-2 & \mathbf{- 1}^{T} \\
\hline 0 & 0 & \mathbf{- 1} & \mathbf{- 1} & \hat{L}+2 I
\end{array}\right]
$$


where $\hat{L}$ is the Laplacian matrix for the subgraph of $G$ induced by deleting the vertices of degree 1 and the vertices of degree $n-2$. By considering eigenvectors of $L$ of the form $\left[\begin{array}{c}a \\ a \\ b \\ b \\ c \mathbf{1}\end{array}\right]$ and $\left[\begin{array}{c}a \\ -a \\ b \\ -b \\ \hline 0\end{array}\right]$, we find that the eigenvalues of the following two matrices are also eigenvalues of $L$ :

$$
M_{1}=\left[\begin{array}{ccc}
1 & -1 & 0 \\
-1 & n-3 & -(n-4) \\
0 & -2 & 2
\end{array}\right], M_{2}=\left[\begin{array}{cc}
1 & -1 \\
-1 & n-1
\end{array}\right]
$$

The eigenvalues of $M_{1}$ are $0, \frac{n \pm \sqrt{n^{2}-4 n}}{2}$, while those of $M_{2}$ are $\frac{n \pm \sqrt{n^{2}-4 n+8}}{2}$. In particular, since $n \geq 5, L$ must have an eigenvalue $\frac{n-\sqrt{n^{2}-4 n+8}}{2}$ in the interval $(1,2)$, and another eigenvalue $\frac{n-\sqrt{n^{2}-4 n}}{2}$ in the interval $(2,3)$, thus contradicting Lemma 3.4. We conclude that $n$ must be 4 and $G$ must be $P_{4}$.

Our next two lemmas rule out certain structures in an NT graph.

Lemma 3.6 Let $G$ be a connected graph $G$ on $n \geq 4$ vertices with minimum degree 1, maximum degree $n-2$, $p$ vertices of degree 1 and $q$ vertices of degree $n-2$. Suppose that $q=1, p \geq 2$ and that one of the pendant vertices is not adjacent to the vertex of degree $n-2$. Then $G$ is not an NT graph.

Proof: Suppose, to the contrary, that $G$ is an NT graph. We label the vertex of degree $n-2$ by $u$. Suppose that the single pendant vertex not adjacent to $u$ is adjacent to vertex $w$. Let $C_{1}, \ldots, C_{r}$ denote the connected components at $u$ that do not consist of a single vertex; see Figure 1.

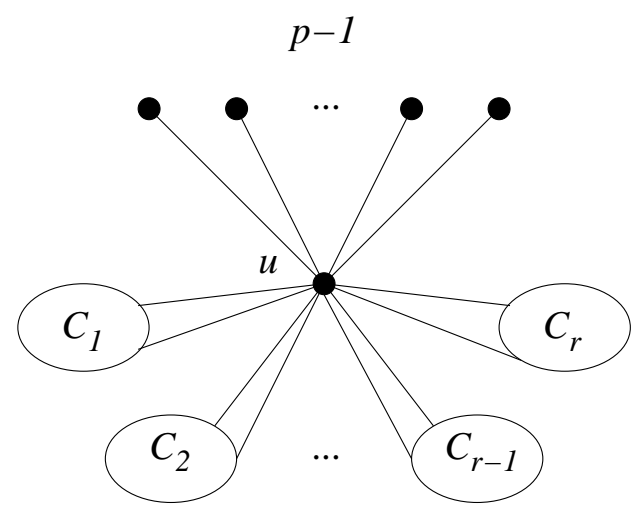

Figure 1: Connected components at $u$ 
Note that $d_{1}^{*}=n, d_{2}^{*}=n-p$ and $d_{n-2}^{*}=1$. Let $L$ be the Laplacian matrix for $G$. For each $i=1, \ldots, r$, denote the spectral radius of the principal submatrix of $L$ corresponding to the vertices of $C_{i}$ by $\rho\left(L\left(C_{i}\right)\right.$ ). Applying interlacing (to the submatrix of $L$ formed by deleting the row and column corresponding to $u$ ), we find that $n-p=\lambda_{n-1}(G) \leq$ $1+\max \left\{\rho\left(L\left(C_{i}\right)\right) \mid i=1, \ldots, r\right\} \leq 1+\max \left\{\mid C_{i} \| i=1, \ldots, r\right\}$. In particular, some $C_{i}$ contains at least $n-p-1$ vertices. Thus we find that $r=1$ and that $\left|C_{1}\right|=n-p$ (for if $r \geq 2$, then necessarily $r=2$ and $\left|C_{1}\right|=n-p-1$, and then there are $p+1$ pendant vertices, contrary to assumption).

If we have $n-p=2$, then we see that $G$ is the 'broom' depicted in Figure 2, which is readily determined not to be an NT graph. We suppose henceforth that $n-p \geq 3$.

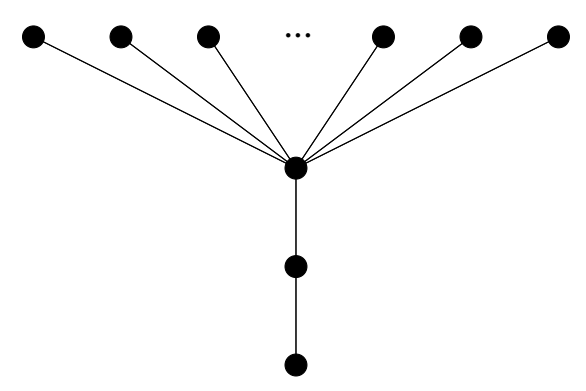

Figure 2: The broom

Denote the pendant vertex adjacent to $w$ by $v$, and let $\tilde{L}$ denote the Laplacian matrix for the connected graph $C_{1} \backslash\{v\}$. Then $L(G)$ can be written as

$$
L=\left[\begin{array}{cccc}
I & -\mathbf{1} & 0 & 0 \\
-\mathbf{1}^{T} & n-2 & -\mathbf{1}^{T} & 0 \\
0 & -\mathbf{1} & I+\tilde{L}+e_{w} e_{w}^{T} & -e_{w} \\
0 & 0 & -e_{w}^{T} & 1
\end{array}\right]
$$

(Here vertex $v$ corresponds to the last row and column of $L$, and $e_{w}$ has a single 1 in the position corresponding to vertex $w$.) It follows that

$$
\operatorname{rank}(L-I)=\operatorname{rank}\left(\left[\begin{array}{cccc}
0 & -1 & 0 & 0 \\
-1 & n-3 & -\mathbf{1}^{T} & 0 \\
0 & -\mathbf{1} & \tilde{L}+e_{w} e_{w}^{T} & -e_{w} \\
0 & 0 & -e_{w} & 0
\end{array}\right]\right)=
$$

$\operatorname{rank}\left(\left[\begin{array}{cccc}0 & -1 & 0 & 0 \\ -1 & 0 & -0^{T} & 0 \\ 0 & 0 & \tilde{L}+e_{w} e_{w}^{T} & -e_{w} \\ 0 & 0 & -e_{w}^{T} & 0\end{array}\right]\right)=2+\operatorname{rank}\left(\left[\begin{array}{cc}\tilde{L}+e_{w} e_{w}^{T} & -e_{w} \\ -e_{w}^{T} & 0\end{array}\right]\right)$.

Note that if $\left[\begin{array}{cc}\tilde{L}+e_{w} e_{w}^{T} & -e_{w} \\ -e_{w}^{T} & 0\end{array}\right]\left[\begin{array}{l}x \\ y\end{array}\right]=0$, then $\tilde{L} x=y e_{w}, e_{w}^{T} x=0$, and hence $x^{T} \tilde{L} x=0$. Since $C_{1} \backslash\{v\}$ is connected, it follows that $x$ is a multiple of $\mathbf{1}$. As $e_{w}^{T} x=0$, we see that $x=0$, and hence $y=0$. We conclude that $\operatorname{rank}(L-I)=2+n-p$, so that 1 is 
an eigenvalue of $L$ of multiplicity $p-2$. Hence we have $d_{n-2}^{*}=1, d_{n-3}^{*}=1, \ldots, d_{n-p+1}^{*}=1$ and $d_{n-p}^{*} \geq 2$. In particular, we must have $p \geq 3$.

Observe that if $a \neq u, w$ is a vertex of $G$, then the degree of $a$ is at most $n-p-1$. Hence $d_{n-p}^{*} \leq 2$, so that in fact $d_{n-p}^{*}=2$, and $w$ has degree $n-p$. Let $\hat{G}$ be the subgraph of $G$ formed by deleting $u, w$, and all pendant vertices. Then we may write

$$
L=\left[\begin{array}{c|c|c|c|c}
I & -\mathbf{1} & 0 & 0 & 0 \\
\hline-\mathbf{1}^{T} & n-2 & -\mathbf{1}^{T} & -1 & 0 \\
\hline 0 & -\mathbf{1} & L(\hat{G})+2 I & -\mathbf{1} & 0 \\
\hline 0 & -1 & -\mathbf{1}^{T} & n-p & -1 \\
\hline 0 & 0 & 0 & -1 & 1
\end{array}\right]
$$

so that the eigenvalues of $L$ are: $\lambda(\hat{G})+2$ for each eigenvalue $\lambda(\hat{G})$ with an eigenvector orthogonal to $\mathbf{1}, 1^{(p-2)}$, and the eigenvalues of the matrix

$$
M=\left[\begin{array}{ccccc}
1 & -1 & 0 & 0 & 0 \\
-(p-1) & n-2 & -(n-p-2) & -1 & 0 \\
0 & -1 & 2 & -1 & 0 \\
0 & -1 & -(n-p-2) & n-p & -1 \\
0 & 0 & 0 & -1 & 1
\end{array}\right]
$$

Recall that $L$ has $n-p$ and 2 as eigenvalues. Note that

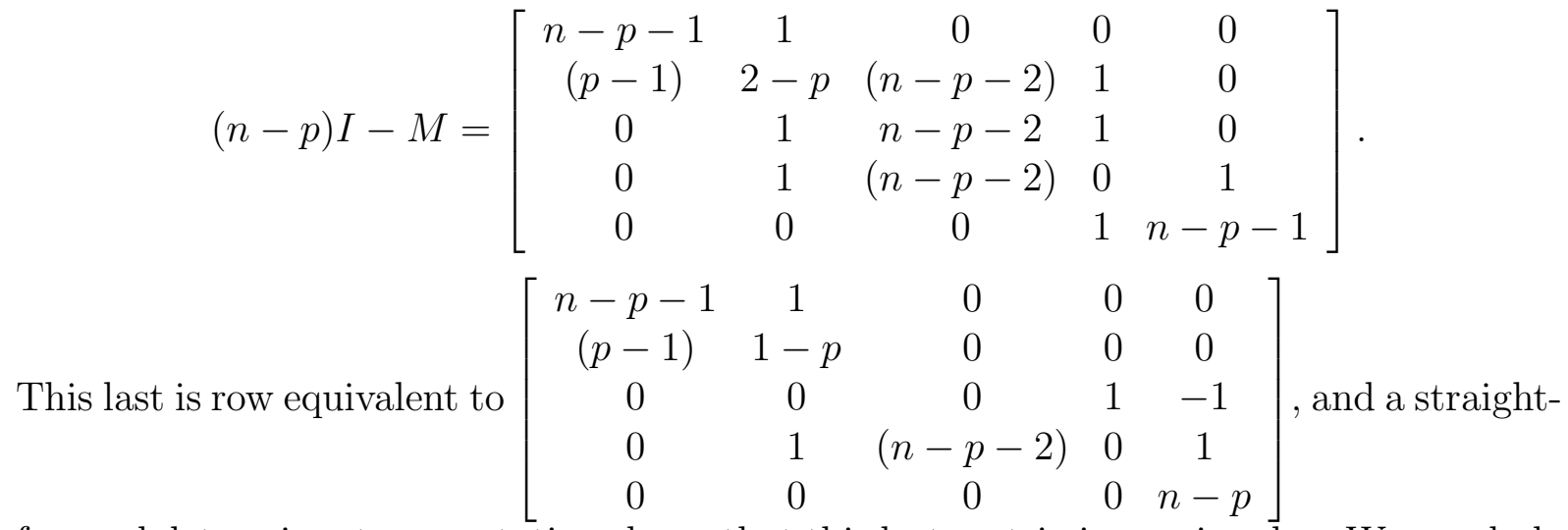
forward determinant computation shows that this last matrix is nonsingular. We conclude that $n-p-2$ must be an eigenvalue of $L(\hat{G})$, so that $\hat{G}$ is a join.

$$
\text { Note also that } 2 I-M=\left[\begin{array}{ccccc}
1 & 1 & 0 & 0 & 0 \\
(p-1) & 4-n & (n-p-2) & 1 & 0 \\
0 & 1 & 0 & 1 & 0 \\
0 & 1 & (n-p-2) & p+2-n & 1 \\
0 & 0 & 0 & 1 & 1
\end{array}\right] \text {, which is row }
$$

and column equivalent to the matrix

$$
\left[\begin{array}{ccccc}
1 & 0 & 0 & 0 & 0 \\
(p-1) & 5-n-p & (n-p-2) & 1 & 0 \\
0 & 1 & 0 & 1 & 0 \\
0 & 0 & (n-p-2) & p-n & 1 \\
0 & 0 & 0 & 0 & 1
\end{array}\right]
$$


It now follows that $\operatorname{det}(2 I-M)=(n-p-2)(2 n-4)>0$. Thus 2 is not an eigenvalue of $M$, so we find that $L(\hat{G})$ must have a null vector that is orthogonal to $\mathbf{1}$. We conclude then that $\hat{G}$ is disconnected. Hence $\hat{G}$ is both disconnected and a join of graphs, a contradiction.

Consequently, $G$ cannot be an NT graph.

Lemma 3.7 Suppose that $G$ is a connected graph $G$ on $n \geq 4$ vertices with minimum degree 1 , maximum degree $n-2, p=1$ vertex of degree 1 and $q$ vertices of degree $n-2$. Suppose that the pendant vertex is adjacent to a vertex $u$ of degree $n-2$. Then $G$ is not an NT graph.

Proof: Suppose first that $q \geq 2$. Consider $\bar{G}$, which has minimum degree 1 , maximum degree $n-2$, and $q$ pendant vertices. From Theorem 2.3, any disconnected NT graph has maximum degree at most $n-3$, so if $\bar{G}$ is not connected, then it is not an NT graph, and hence neither is $G$. In the case that $\bar{G}$ is connected, note that necessarily in $\bar{G}$, there is a pendant vertex $u$ is not adjacent to the unique vertex of degree $n-2$. By Lemma 3.6, $\bar{G}$ is not an NT graph, and hence neither is $G$.

It remains only to consider the case that $q=1$. Suppose to the contrary that $G$ is an NT graph. We have $d_{1}^{*}=n, d_{2}^{*}=n-1, d_{n-2}^{*}=1$ so in particular, 1 is an eigenvalue. Let $w$ be the vertex of $G$ not adjacent to $u$. Let $\tilde{L}$ be the Laplacian matrix for the subgraph $\tilde{G}$ induced by deleting the pendant vertex and $u$. We have

$$
L=\left[\begin{array}{cc|c}
1 & -1 & 0^{T} \\
-1 & n-2 & -\mathbf{1}^{T}+e_{w} \\
\hline 0 & -\mathbf{1}+e_{w} & \tilde{L}+I-e_{w} e_{w}^{T}
\end{array}\right]
$$

Suppose that the vector $\left[\begin{array}{l}a \\ b \\ x\end{array}\right]$ is an eigenvector corresponding to the eigenvalue 1 . We then find from the eigenequation that $b=0$ and $e_{w}^{T} x=0$ (the latter since $a+b+\mathbf{1}^{T} x=0$ ), and hence $\tilde{L} x=0$. Since $\tilde{L}$ has a null vector orthogonal to $e_{w}$, we conclude that $\tilde{G}$ is disconnected, say with $r \geq 2$ components, $C_{1}, \ldots, C_{r}$, each necessarily having at least two vertices (since $p=1$ ). Further, it follows that the multiplicity of 1 as an eigenvalue of $L$ is the same as the number of linearly independent null vectors for $\tilde{L}$ that are orthogonal to $e_{w}$; that number is $r-1$. As a result, we have $d_{n-2}^{*}=1, d_{n-3}^{*}=1, \ldots, d_{n-r}^{*}=1$, and $d_{n-r-1}^{*} \geq 2$. Note that any vertex in $C_{i}$ has degree at most $1+\left|C_{i}\right|-1=\left|C_{i}\right|=$ $n-2-\sum_{j \neq i}\left|C_{j}\right| \leq n-2-2(r-1)=n-2 r$. As $n-2 r<n-r-1$, it follows that $d_{n-r-1}^{*}=1$, a contradiction.

We conclude then that $G$ cannot be an NT graph.

Our next two results describe certain constructions that yield NT graphs. 
Proposition 3.8 Let $G$ be a connected graph $G$ on $n \geq 4$ vertices with minimum degree 1 , maximum degree $n-2, p$ vertices of degree 1 and $q$ vertices of degree $n-2$. Suppose that $q=1, p \geq 2$ and all of the pendant vertices are adjacent to the vertex of degree $n-2$, which we denote by $u$. Then $G$ is an NT graph if and only if $n$ is even, and $G$ is the graph $N T_{1}(n)$ depicted in Figure 3.

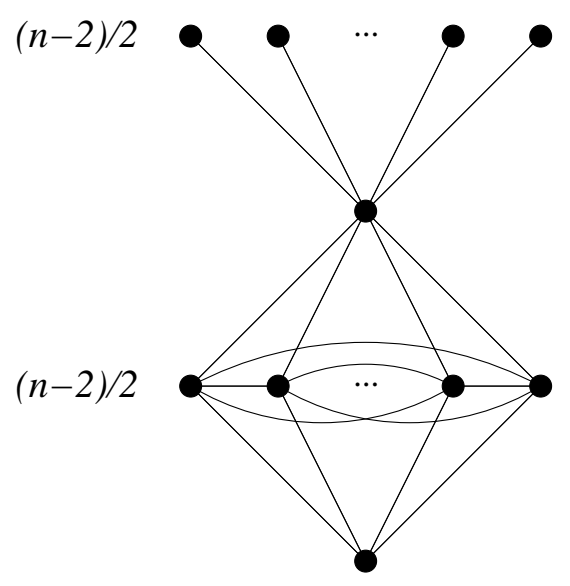

$N T_{1}(n)$

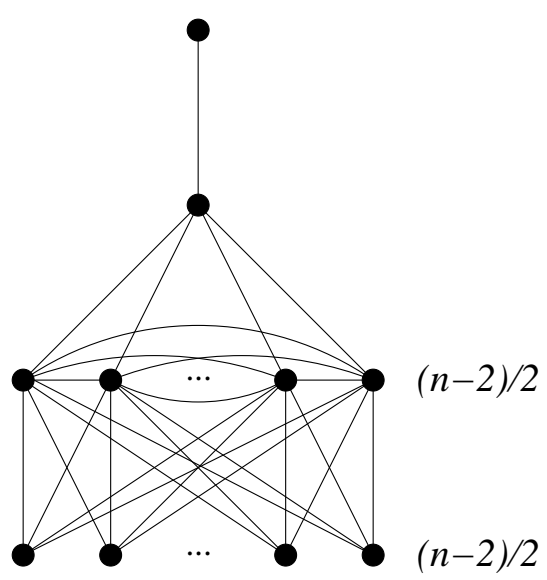

$N T_{2}(n)$

Figure 3: Two NT graphs

Proof: Suppose that $G$ is an NT graph, and denote the connected components at $u$ that have at least two vertices by $C_{1}, \ldots, C_{r}$. Applying interlacing to the principal submatrix of $L(G)$ obtained by deleting the row and column corresponding to $u$, we have $\lambda_{n-1}=$ $d_{2}^{*}=n-p \leq 1+\max \left\{\rho\left(L\left(C_{i}\right)\right) \mid i=1, \ldots, r\right\} \leq 1+\max \left\{\mid C_{i} \| i=1, \ldots, r\right\} \leq n-p$. It follows that $r=1,\left|C_{1}\right|=n-p-1$, and $\rho\left(L\left(C_{1}\right)\right)=n-p-1$. Hence $C_{1}$ is a join, say of $l \geq 2$ graphs $G_{1}, \ldots, G_{l}$, each of which is either disconnected, or consists of a single vertex, and where the unique vertex, say $w$, of $G$ that is not adjacent to $u$ is in $G_{l}$. Set $G_{0}=\vee_{i=1}^{l-1} G_{i}$, and suppose that $\left|G_{l}\right|=n_{l}$.

We write $L$ as

$$
\begin{aligned}
& \qquad L=\left[\begin{array}{c|c|c|c}
I_{p} & -\mathbf{1} & 0 & 0 \\
\hline-\mathbf{1}^{T} & n-2 & -\mathbf{1}^{T} & -\mathbf{1}^{T}+e_{w}^{T} \\
\hline 0 & -\mathbf{1} & L\left(G_{0}\right)+\left(n_{l}+1\right) I & -J \\
\hline 0 & -\mathbf{1}+e_{w} & -J & L\left(G_{l}\right)+\left(n-p-n_{l}\right) I-e_{w} e_{w}^{T}
\end{array}\right] . \\
& \text { By considering eigenvectors of the form }\left[\begin{array}{c}
\frac{x}{0} \\
\frac{0}{0}
\end{array}\right],\left[\begin{array}{c}
\frac{0}{0} \\
\frac{y}{0}
\end{array}\right] \text {, where each of } x \text { and } y \text { sums to }
\end{aligned}
$$


0 , and eigenvectors of the form $\left[\frac{\frac{a \mathbf{1}}{b}}{\frac{c \mathbf{1}}{d}}\right]$, it follows that the eigenvalues of $L$ consist of $1^{(p-1)}, \lambda+n_{l}+1$ for each eigenvalue $\lambda$ of $G_{0}$ with an eigenvector orthogonal to $\mathbf{1}$, as well as the eigenvalues of the matrix

$$
M=\left[\begin{array}{ccc|c}
1 & -1 & 0 & 0^{T} \\
-p & n-2 & -\left(n-p-1-n_{l}\right) & -\mathbf{1}^{T}+e_{w}^{T} \\
0 & -1 & n_{l}+1 & -\mathbf{1}^{T} \\
\hline 0 & -\mathbf{1}+e_{w} & -\left(n-p-1-n_{l}\right) \mathbf{1} & L\left(G_{l}\right)+\left(n-p-n_{l}\right) I-e_{w} e_{w}^{T}
\end{array}\right]
$$

Note that $(n-p) I-M$ is row equivalent to

$$
\left[\begin{array}{ccc|c}
n-p-1 & 1 & 0 & 0^{T} \\
p & 1-p & 0 & -e_{w}^{T} \\
0 & 1 & n-p-1-n_{l} & \mathbf{1}^{T} \\
\hline 0 & -e_{w} & 0 & n_{l} I-L\left(G_{l}\right)-J+e_{w} e_{w}^{T}
\end{array}\right]
$$

it follows that $n-p$ is an eigenvalue of $M$ if and only if the matrix

$$
\tilde{M}=\left[\begin{array}{ccc}
n-p-1 & 1 & 0^{T} \\
p & 1-p & -e_{w}^{T} \\
0 & -e_{w} & \left(n_{l} I-L\left(G_{l}\right)-J+e_{w} e_{w}^{T}\right)
\end{array}\right]
$$

is singular. If it happens that $n_{l}=1$, it is readily established that $\tilde{M}$ is nonsingular.

Suppose that $n_{l} \geq 2$ and that $\tilde{M}\left[\begin{array}{l}a \\ b \\ x\end{array}\right]=0$. Then $b=-a(n-p-1)$, so that $p a+(p-1)(n-p-1) a=e_{w}^{T} x$ and $a(n-p-1) e_{w}+\left(n_{l} I-L\left(G_{l}\right)-J+e_{w} e_{w}^{T}\right) x=0$. Thus, $x^{T}\left(n_{l} I-L\left(G_{l}\right)-J+e_{w} e_{w}^{T}\right) x+a(n-p-1)(p a+(p-1)(n-p-1) a)=0$. Noting that $n_{l} I-L\left(G_{l}\right)-J+e_{w} e_{w}^{T}$ is a positive semidefinite matrix, we have $a=0$ and $x^{T}\left(n_{l} I-L\left(G_{l}\right)-J+e_{w} e_{w}^{T}\right) x=0$. But $n_{l} I-L\left(G_{l}\right)-J$ is the Laplacian matrix for $\overline{G_{l}}$, which is connected. Hence, $x$ must be 0 , and we conclude that $\tilde{M}$ is nonsingular.

Consequently, the multiplicity of $n-p$ as an eigenvalue of $L$ coincides with the number of linearly independent eigenvectors of $G_{0}$ that are orthogonal to $\mathbf{1}$ and correspond to the eigenvalue $n-p-1-n_{l}$ of $G_{0}$. That multiplicity is $l-2$. Hence we have $d_{2}^{*}=n-p, d_{3}^{*}=$ $n-p, \ldots, d_{l-1}^{*}=n-p, d_{l}^{*} \leq n-p-1$.

Observe that each vertex in $G_{0} \cup\left(G_{l} \backslash\{w\}\right)$ has degree (in $G$ ) at least $l$, as does vertex $u$. Thus $d_{l}^{*} \geq n-p-1$, and we find that in fact $d_{l}^{*}$ must equal $n-p-1$. Hence, vertex $w$ has degree at most $l-1$, which happens only if each of $G_{1}, \ldots, G_{l-1}$ is an isolated vertex, and vertex $w$ is isolated in $G_{l}$. Write $G_{l}=\hat{G} \cup\{w\}$. Suppose that $n-p-l-1 \geq 1$. We 
have

$$
L=\left[\begin{array}{ccccc}
I & \mathbf{- 1} & 0 & 0 & 0 \\
-\mathbf{1}^{T} & n-2 & -\mathbf{1}^{T} & -\mathbf{1}^{T} & 0 \\
0 & -\mathbf{1} & (n-p) I-J & -J & -\mathbf{1} \\
0 & -\mathbf{1} & -J & L(\hat{G})+l I & 0 \\
0 & 0 & -\mathbf{1}^{T} & 0 & l-1
\end{array}\right]
$$

we find that each eigenvalue of the matrix

$$
M_{0}=\left[\begin{array}{ccccc}
1 & -1 & 0 & 0 & 0 \\
-p & n-2 & -(l-1) & -(n-p-l-1) & 0 \\
0 & -1 & n-p-l+1 & -(n-p-l-1) & -1 \\
0 & -1 & -(l-1) & l & 0 \\
0 & 0 & -(l-1) & 0 & l-1
\end{array}\right]
$$

is necessarily an eigenvalue of $L$. Uninteresting computations reveal that $\operatorname{det}((n-p) I-$ $\left.M_{0}\right)=-p(n-p)(l-1)<0$, while $\operatorname{det}\left((n-p-1) I-M_{0}\right)=p(n-p-l-1)(n-p-1)^{2}>0$. Hence $M_{0}$ has a noninteger eigenvalue in the interval $(n-p-1, n-p)$, and thus so does $L$, a contradiction. We conclude then that $n-p-l-1=0$.

Thus we have

$$
L=\left[\begin{array}{cccc}
I & -\mathbf{1} & 0 & 0 \\
-\mathbf{1}^{T} & n-2 & -\mathbf{1}^{T} & 0 \\
0 & -\mathbf{1} & (n-p) I-J & -\mathbf{1} \\
0 & 0 & -\mathbf{1}^{T} & n-p-2
\end{array}\right]
$$

as above, each eigenvalue of the matrix

$$
M_{1}=\left[\begin{array}{cccc}
1 & -1 & 0 & 0 \\
-p & n-2 & -(n-p-2) & 0 \\
0 & -1 & 2 & -1 \\
0 & 0 & -(n-p-2) & n-p-2
\end{array}\right]
$$

is necessarily an eigenvalue of $L$. We find that $\operatorname{det}\left(z I-M_{1}\right)=z((z-1)(z-(n-2))(z-$ $(n+p))-p(n-p-2)-p(z-(n-p)))$. Since the graph $G$ is an NT graph, we see that $d_{l}^{*}=n-p-1$ must be an eigenvalue of $M_{1}$, which occurs only if $p=\frac{n-2}{2}$. In that case, the graph $G$ is readily seen to be the same as $N T_{1}(n)$ in Figure 3.

Conversely, if $G=N T_{1}(n)$, a direct computation reveals it to be an NT graph.

Proposition 3.9 Let $G$ be a connected graph $G$ on $n \geq 4$ vertices with minimum degree 1 , maximum degree $n-2, p=1$ vertex of degree 1 and $q$ vertices of degree $n-2$. Suppose that the pendant vertex is not adjacent to any vertex of degree $n-2$. Then $G$ is an NT graph if and only if $n$ is even, and $G$ is the graph $N T_{2}(n)$ depicted in Figure 3. 
Proof: Suppose that $G$ is an NT graph. Observe that if $q=1$, then by Lemma $3.7, \bar{G}$ is not an NT graph. Hence, $G$ itself is not an NT graph. Consequently, it must be the case that $q \geq 2$.

We may write the corresponding Laplacian matrix as

$$
L=\left[\begin{array}{c|c|c}
(n-1) I-J & -J & 0 \\
\hline-J & L(\hat{G})+q I+e_{j} e_{j}^{T} & -e_{j} \\
\hline 0^{T} & -e_{j}^{T} & 1
\end{array}\right]
$$

where $\hat{G}$ is the subgraph of $G$ induced by the vertices of degrees in $[2, n-3]$. (Here we have taken the last row and column to correspond to the pendant vertex, and we take $j$ to be the vertex adjacent to the pendant vertex.) The eigenvalues of $L$ are $(n-1)^{(q-1)}$, along with those of the matrix

$$
M=\left[\begin{array}{ccc}
(n-q-1) & -\mathbf{1}^{T} & 0 \\
-q \mathbf{1} & L(\hat{G})+q I+e_{j} e_{j}^{T} & -e_{j} \\
0^{T} & -e_{j}^{T} & 1
\end{array}\right]
$$

Since $d_{n-2}^{*}=q<n-1$, we see that $M-q I$ must be singular. Let $\left[\begin{array}{l}a \\ x \\ b\end{array}\right]$ be a corresponding null vector. Then $(n-2 q-1) a=\mathbf{1}^{T} x, L(\hat{G}) x+\left(e_{j}^{T} x\right) e_{j}-b e_{j}-a q \mathbf{1}=0$, and $(1-q) b=e_{j}^{T} x$. Hence $L(\hat{G}) x=a q \mathbf{1}+b q e_{j}$; considering $\mathbf{1}^{T} L(\hat{G}) x=0$ now shows that $b=-a(n-q-1)$. It now follows that $x^{T} L(\hat{G}) x=q a^{2}\left(n-2 q-1-(q-1)(n-q-1)^{2}\right)$.

Since $q \geq 2$, we have $n-2 q-1-(q-1)(n-q-1)^{2}<0$, so that necessarily $a=0, b=0, \mathbf{1}^{T} x=0, e_{j}^{T} x=0$ and $L(\hat{G}) x=0$. Hence, $\hat{G}$ is disconnected, with say $c \geq 2$ components, and the multiplicity of $q$ as an eigenvalue of $L$ is thus equal to the dimension of the subspace of null vectors of $L(\hat{G})$ that are orthogonal to both $\mathbf{1}$ and $e_{j}$. Evidently, that dimension is $c-2$. Consequently, $d_{n-2}^{*}=q, d_{n-3}^{*}=q, \ldots, d_{n-c+1}^{*}=q$, and $d_{n-c}^{*} \geq q+1$. Write $\hat{G}=G_{1} \cup \ldots \cup G_{c}$, with $\left|G_{i}\right|=n_{i}, i=1, \ldots, c$. Note that $n_{1}+\ldots+n_{c}=n-q-1$. Without loss of generality, we take the pendant vertex to be adjacent to $j \in G_{c}$. Note that for each vertex in $G_{i}$ that is distinct from $j$, the degree is at most $q+n_{i}-1=n-2-\sum_{l \neq i} n_{l} \leq n-c-1$; similarly, the degree of vertex $j$ is at most $q+n_{c} \leq n-c$. It now follows that $d_{n-c}^{*} \geq q+1$ only if $n_{l}=1, l=1, \ldots, c-1$, and in addition, vertex $j$ is adjacent to every other vertex in $G_{c}$.

Suppose that $n-q-c-1 \geq 1$. Then we have

$L=\left[\begin{array}{c|c|c|c|c}(n-1) I-J & -J & -J & -\mathbf{1} & 0 \\ \hline-J & q I & 0 & 0 & 0 \\ \hline-J & 0 & L_{c}+(q+1) I & -\mathbf{1} & 0 \\ \hline-\mathbf{1}^{T} & 0 & -\mathbf{1}^{T} & n-c & -1 \\ \hline 0^{T} & 0 & 0^{T} & -1 & 1\end{array}\right]$,

where $L_{c}$ is the Laplacian matrix for the subgraph induced by $G_{c} \backslash\{j\}$. It follows that $L$ has eigenvalues $(n-1)^{(q-1)}, q^{(c-2)}$, and $\lambda+q+1$ for each eigenvalue $\lambda$ of $L_{c}$ orthogonal 
to $\mathbf{1}$, as well as the eigenvalues of the matrix

$$
\bar{M}=\left[\begin{array}{ccccc}
n-q-1 & -(c-1) & -(n-q-c-1) & -1 & 0 \\
-q & q & 0 & 0 & 0 \\
-q & 0 & q+1 & -1 & 0 \\
-q & 0 & -(n-q-c-1) & n-c & -1 \\
0 & 0 & 0 & -1 & 1
\end{array}\right]
$$

We find that $\operatorname{det}(q I-\bar{M})=-q^{2}(c-1)$, while $\operatorname{det}((q+1) I-\bar{M})=q(q+1)(n-q-$ $c-1)(n-q-1)$. Hence $L$ has a noninteger eigenvalue in the open interval $(q, q+1)$, a contradiction.

Consequently, it must be the case that $n-q-c-1=0$, and that

$$
L=\left[\begin{array}{c|c|c|c}
(n-1) I-J & -J & -\mathbf{1} & 0 \\
\hline-J & q I & 0 & 0 \\
\hline-\mathbf{1}^{T} & 0^{T} & q+1 & -1 \\
\hline 0^{T} & 0 & -1 & 1
\end{array}\right]
$$

Since $d_{q+1}^{*}=q+1$, we see that $G$ is an NT graph only if $q+1$ is an eigenvalue of $L$. This last holds only if $\operatorname{det}\left(\left[\begin{array}{cccc}n-2 q-2 & -(n-q-2) & -1 & 0 \\ -q & -1 & 0 & 0 \\ -q & 0 & 0 & -1 \\ 0 & 0 & -1 & -q\end{array}\right]\right)=0$, i.e. only if $(q+1)(n-2 q-2)=0$. Thus $q=\frac{n-2}{2}$, and $G$ is the same as $N T_{2}(n)$ in Figure 3.

The converse is straightforward.

Remark 3.10 Observe that for each even integer $n \geq 4$, the graphs $N T_{1}(n)$ and $N T_{2}(n)$ of Figure 3 are related via complementation. Note also that in the special case that $n=4$, both graphs coincide with $P_{4}$.

Let $\Gamma_{0}=$

$\left\{H \cup K_{2} \mid H\right.$ is a connected threshold graph $\} \cup$

$\left\{H \vee O_{2} \mid H\right.$ is a disconnected threshold graph $\} \cup$

$\left\{N T_{1}(2 n) \mid n \in \mathbb{N}\right\} \cup\left\{N T_{2}(2 n) \mid n \in \mathbb{N}\right\}$.

For each $k \in \mathbb{N}$, let

$$
\Gamma_{k}=\left\{\left(G \vee K_{2}\right) \cup K_{2} \mid H \in \Gamma_{k-1}\right\} \cup\left\{\left(G \cup O_{2}\right) \vee O_{2} \mid H \in \Gamma_{k-1}\right\} .
$$

Here is one of the main results of this section.

Theorem 3.11 A graph $G$ is an NT graph with no isolated vertices and no dominant vertices if and only if $G \in \cup_{k \geq 0} \Gamma_{k}$. 
Proof: Suppose that $G$ is an NT graph on $n \geq 4$ vertices with minimum degree $\delta$ and maximum degree $\Delta$. We claim that $G \in \Gamma_{k}$ for some $k \geq 0$. In order to establish the claim, we proceed by induction on $n$, and note that the case $n=4$ is readily established.

If $G$ is not connected, then by Theorem 2.3, either $G=H \cup K_{2}$ for some connected threshold graph $H$, or $G=\left(K_{2} \vee H_{0}\right) \cup K_{2}$ for some NT graph $H_{0}$ with no dominant or isolated vertices. In the former case, $G \in \Gamma_{0}$; in the latter case, we find from the induction hypothesis that $H_{0} \in \Gamma_{k}$ for some $k$, so that necessarily $G \in \Gamma_{k+1}$.

Suppose next that $G$ is connected. From our discussion at the beginning of this section, it follows that $\Delta=n-2$. If $\delta \geq 2$, we find from Theorem 3.3 that either $G=G_{1} \vee O_{2}$ for some disconnected threshold graph, or $G=\left(O_{2} \cup H\right) \vee O_{2}$ for some NT graph with no dominant or isolated vertices. Hence, either $G \in \Gamma_{0}$, or (applying the induction hypothesis to $H) G \in \Gamma_{k}$ for some $k \in \mathbb{N}$. Finally, we suppose that $\delta=1$. In that case, it follows from Lemmas 3.5,3.6 and 3.7, as well as Propositions 3.8 and 3.9, that $n$ is even, and $G$ is either $N T_{1}(n)$ or $N T_{2}(n)$.

Conversely, a straightforward proof by induction on $k$ shows that for each $k \geq 0$, every graph in $\Gamma_{k}$ is an NT graph.

The following results are immediate from Propositions 2.1 and 3.1.

Corollary 3.12 A graph $G$ is an NT graph with an isolated vertex if and only if $G$ has the form $G=\left(H \vee K_{m_{1}}\right) \cup O_{m_{2}}$ for some $m_{1} \geq 0, m_{2} \geq 1$ and some $H \in \cup_{k \geq 0} \Gamma_{k}$.

Corollary 3.13 A graph $G$ is an NT graph with a dominant vertex if and only if $G$ has the form $G=\left(H \cup O_{m_{1}}\right) \vee K_{m_{2}}$ for some $m_{1} \geq 0, m_{2} \geq 1$ and some $H \in \cup_{k \geq 0} \Gamma_{k}$.

\section{Commentary and consequences}

In this section, we provide some ancillary results and discussion on NT graphs.

Recall that a graph is Laplacian integral if its Laplacian spectrum consists entirely of integers. In light of the fact that an NT graph has at most two noninteger eigenvalues, it is natural to discuss the class of Laplacian integral NT graphs. The following classes of graphs are central to that discussion.

Let $\mathcal{C}_{0}=\left\{H \cup K_{2} \mid H\right.$ is a connected threshold graph $\} \cup$ $\left\{H \vee O_{2} \mid H\right.$ is a disconnected threshold graph $\}$. For each $k \in \mathbb{N}$, let

$$
\mathcal{C}_{k}=\left\{\left(G \vee K_{2}\right) \cup K_{2} \mid H \in \mathcal{C}_{k-1}\right\} \cup\left\{\left(G \cup O_{2}\right) \vee O_{2} \mid H \in \mathcal{C}_{k-1}\right\}
$$

The proof of the following result is parallel to that of Theorem 3.11, Corollary 3.12 and Corollary 3.13, and is omitted.

Theorem 4.1 a) A graph $G$ is a Laplacian integral NT graph with no isolated vertex and no dominant vertex if and only if $G \in \cup_{k \geq 0} \mathcal{C}_{k}$.

b) A graph $G$ is a Laplacian integral NT graph with an isolated vertex if and only if $G$ has the form $G=\left(H \vee K_{m_{1}}\right) \cup O_{m_{2}}$ for some $m_{1} \geq 0, m_{2} \geq 1$ and some $H \in \cup_{k \geq 0} \mathcal{C}_{k}$. 
c) A graph $G$ is an NT graph with a dominant vertex if and only if $G$ has the form $G=\left(H \cup O_{m_{1}}\right) \vee K_{m_{2}}$ for some $m_{1} \geq 0, m_{2} \geq 1$ and some $H \in \cup_{k \geq 0} \mathcal{C}_{k}$.

Recall that the threshold graphs can be characterized in two different ways: as the graphs $G$ such that $\Lambda(G)=D^{*}(G)$, and also as the graphs having no induced subgraphs equal to $P_{4}, C_{4}$, or $2 K_{2}$. In light of that fact, it is natural to wonder whether the property of being an NT graph also places a restriction on the presence of subgraphs equal to one of $P_{4}, C_{4}$, or $2 K_{2}$. The following example addresses that question.

Example 4.2 Observe that each of $C_{4}, 2 K_{2}$ and $P_{4}$ is an example of an NT graph. Further, appealing to Theorem 3.3, we find that $\left(\mathrm{O}_{2} \cup 2 K_{2}\right) \vee O_{2}$ contains both $C_{4}$ and $2 K_{2}$ as induced subgraphs, while $\left(\mathrm{O}_{2} \cup P_{4}\right) \vee O_{2}$ contains both $C_{4}$ and $P_{4}$ as induced subgraphs. Further, $\left(K_{2} \vee P_{4}\right) \cup K_{2}$ contains both $P_{4}$ and $2 K_{2}$ as induced subgraphs. Finally we also note that $\left.\left(O_{2} \cup\left(K_{2} \vee P_{4}\right) \cup K_{2}\right)\right) \vee O_{2}$ contains each of $P_{4}, C_{4}$, and $2 K_{2}$ as induced subgraphs. Hence, we see that an NT graph may contain as induced subgraphs either one, two or all three of the graphs $P_{4}, C_{4}$, and $2 K_{2}$.

In view of our discussion of the Grone-Merris conjecture in Section 1, the following result is a natural one.

Theorem 4.3 If $G$ is any NT graph, then $D^{*}(G) \succeq \Lambda(G)$.

Proof: We will show by induction on $k$ that for each $k \geq 0$ and each $G \in \Gamma_{k}, D^{*}(G) \succeq \Lambda(G)$. Throughout the proof, we will use the fact that if $D^{*}(G) \succeq \Lambda(G)$ for some graph $G$, then necessarily $D^{*}(\bar{G}) \succeq \Lambda(\bar{G})$.

Suppose that $k=0$. Observe that for $G=N T_{1}(n)$, we have $d_{n}^{*}(G)=n>\lambda_{n}(G)=$ $\frac{n+\sqrt{n^{2}-4 n+8}}{2}, \lambda_{n-i+1}=d_{i}^{*}, i=2, \ldots, n-2, \lambda_{2}=\frac{n-\sqrt{n^{2}-4 n+8}}{2}$, and $\lambda_{1}=d_{n-1}^{*}=d_{n}^{*}=0$. It follows readily then that $D^{*}\left(N T_{1}(n)\right) \succeq \Lambda\left(N T_{1}(n)\right)$. As $N T_{2}(n)=\overline{N T_{1}(n)}$, we find that $D^{*}\left(\overline{N T_{1}(n)}\right) \succeq \Lambda\left(\overline{N T_{1}(n)}\right)$ as well. Similarly, for a connected threshold graph $H$ on $n-2$ vertices, let $G=H \cup K_{2}$. Then $\Lambda(G)=\left\{0^{(2)}, d_{1}^{*}(H), \ldots, d_{n-2}^{*}(H), 2\right\}$, while $D^{*}(G)=\left\{n, d_{2}^{*}(H), \ldots, d_{n-2}^{*}(H), 0^{(2)}\right\}$, and it follows that $D^{*}(G) \succeq \Lambda(G)$. Considering $\bar{G}$, we find also that for any disconnected threshold graph $H, D^{*}\left(H \vee O_{2}\right) \succeq \Lambda\left(H \vee O_{2}\right)$. Hence, each graph in $\Gamma_{0}$ has the desired property.

Next, suppose that we have an NT graph $H \in \Gamma_{k}$ on $n-4$ vertices with no dominant vertex and no isolated vertex, and consider the graph $G=\left(K_{2} \vee H\right) \cup K_{2}$. Then $\Lambda(G)=\left\{(n-2)^{(2)}, \lambda_{n-4}(H)+2, \lambda_{n-5}(H)+2, \ldots, \lambda_{2}(H)+2,2,0^{(2)}\right\}$, while $D^{*}(G)=$ $\left\{n, n-2, d_{1}^{*}(H)+2, d_{2}^{*}(H)+2, \ldots, d_{n-5}^{*}(H)+2,0^{(3)}\right\}$. From the induction hypothesis applied to $H$, it now follows that $D^{*}(G) \succeq \Lambda(G)$, and hence that $D^{*}(\bar{G}) \succeq \Lambda(\bar{G})$ as well. We then deduce that each graph in $\Gamma_{k+1}$ satisfies the desired majorization inequality.

Remark 4.4 As note in section 1 , we have $x \succeq y$ for vectors $x$ and $y$ if and only if there is a symmetric matrix, $M(x, y)$ say, with spectrum $x$ and diagonal $y$. Adopting that viewpoint, we see the main result of [9] characterizes the graphs $G$ such that $M\left(D^{*}(G), \Lambda(G)\right)$ 
can be taken to be a diagonal matrix. In a similar manner, we see that if $G$ is an NT graph on $n$ vertices, then $M\left(D^{*}(G), \Lambda(G)\right)$ can be taken to be a direct sum of one $2 \times 2$ block, and $(n-2) 1 \times 1$ blocks.

We conclude this paper with some open problems, and suggestions for future research. 1. Is there a characterization of NT graphs in terms of forbidden subgraphs? In view of Example 4.2, if such a characterization exists, it must involve subgraphs on more than four vertices.

2. Characterize, construct, or describe the graphs $G$ such that the symmetric matrix $M\left(D^{*}(G), \Lambda(G)\right)$ of Remark 4.4 can be taken to be a direct sum of $1 \times 1$ and $2 \times 2$ matrices. In order to make the problem more tractable, it may be helpful to restrict the number of $2 \times 2$ blocks.

3. Characterize, construct, or describe the graphs $G$ on $n$ vertices such that $D^{*}(G)$ and $\Lambda(G)$ agree in $n-3$ places.

4. Consider problems 2 and 3 above, but impose the additional constraint that the graphs under consideration are Laplacian integral.

\section{References}

[1] R. Bapat, A. Lal and S. Pati, Laplacian spectrum of weakly quasi-threshold graphs, Graphs and Combinatorics, to appear.

[2] H. Christianson and V. Reiner, The critical group of a threshold graph, Linear and its Applications 349 (2002), 233-244.

[3] D. Corneil, H. Lerch and L. Stewart Burlingham, Complement reducible graphs, Discrete Applied Mathematics 3 (1981), 163-174.

[4] R. Grone and R. Merris, The Laplacian spectrum of a graph II, SIAM Journal on Discrete Mathematics 7 (1994), 221-229.

[5] P. Hammer and A. Kelmans, Laplacian spectra and spanning trees in threshold graphs, Discrete Applied Mathematics 65 (1996), 255-273.

[6] R. Horn and C. Johnson, Matrix Analysis, Cambridge University Press, New York, 1985.

[7] S. Kirkland, A bound on the algebraic connectivity of a graph in terms of the number of cutpoints, Linear and Multilinear Algebra 47 (2000), 93-103.

[8] S. Kirkland, Constructably Laplacian Integral Graphs, Linear Algebra and its Applications 423 (2007), 3-21.

[9] R. Merris, Degree maximal graphs are Laplacian integral, Linear Algebra and its Applications 199 (1994), 381-389.

[10] R. Merris, Laplacian matrices of graphs: a survey, Linear Algebra and its Applications 197, 198 (1994), 143-176.

[11] R. Merris, Laplacian graph eigenvectors, Linear Algebra and its Applications, 278 (1998), 221-236. 
[12] R. Merris, Graph Theory, Wiley, New York, 2001.

[13] B. Mohar, Laplace eigenvalues of graphs-a survey, Discrete Mathematics 109 (1992), 171-183.

[14] T. Stephen, A majorization bound for the eigenvalues of some graph Laplacians, SIAM Journal on Discrete Mathematics, 21 (2007), 303-312. 